\title{
EL CUIDADO DE LA FIBROMIALGIA EN LA MUJER Y LA IMPORTANCIA DE LA PRÁCTICA DEPORTIVA
}

\author{
CARING FOR FIBROMYALGIA \\ IN WOMEN AND THE \\ IMPORTANCE OF SPORT
}

Recibido mayo 2016 Aceptado enero 2017

Correspondencia: Dra. en C. Adela Alba Leonel Antiguo camino a Xochimilco y Viaducto Tlalpan Col. San Lorenzo Huipulco C. P. 14370 Tlalpan, México, Ciudad de México Tel. 55562332 Ext. 269 mail: Adelaalbaleonel65@gmail.com

\section{Autores:}

M en C. Julio Hernández Falcón

Mtro. en Investigación de Servicios de Salud Profesor de Carrera Asociado "C", Escuela Nacional de Enfermería y Obstetricia, UNAM.

Dra. Adela Alba Leonel

Dra. en Ciencias Salud Campo Disciplinar Epidemiología. Profesor de Carrera Asociado "C", Escuela Nacional de Enfermería y Obstetricia, UNAM.

Palabras clave: fibromialgia, deporte y fibromialgia, fibromialgia y cuidados, fibromialgia y mujer

Key words: fibromyalgia, sports and fibromyalgia, fibromyalgia and care, fibromyalgia and woman. 
RESUMEN

Introducción: la fibromialgia suele agravarse con el esfuerzo físico, la fatiga, la humedad ambiental, cambios climáticos e incluso por algunos factores laborales que tiene que ver con la postura durante el trabajo.

Objetivo: conocer si la práctica deportiva es una estrategia viable para disminuir las manifestaciones clínicas de la fibromialgia.

Metodología: se realizó una revisión sistemática, tomando como palabras claves fibromialgia, deporte, cuidado y mujer, en los últimos diez años

Resultados: existen evidencias del efecto positivo de la práctica deportiva, del ejercicio aeróbico en las manifestaciones de la fibromialgia, además de ser un efecto positivo en el estado de ánimo relacionado con la incidencia de fibromialgia.

Conclusiones: la práctica deportiva es una estrategia viable para disminuir las manifestaciones clínicas de la fibromialgia

Palabras clave: fibromialgia, deportey fibromialgia, fibromialgia y cuidados, fibromialgia y mujer.
ABSTRACT

Background: fibromyalgia is often aggravated by exertion, fatigue, humidity, climate change and even some workplace factors that has to do with posture during work.

Objective: to determine whether the sport is a viable strategy to reduce the clinical manifestations of fibromyalgia.

Methodology: a systematic review was conducted using as keywords fibromyalgia, sport, care and women, in the last ten years

Results: there is evidence of the positive effect of sports, aerobic exercise in manifestations of fibromyalgia, besides being a positive effect on mood related to the incidence of fibromyalgia.

Conclusions: playing sports is a viable strategy to reduce the clinical manifestations of fibromyalgia.

Keywords: fibromyalgia, sports and fibromyalgia, fibromyalgia and care, fibromyalgia and woman. 


\section{INTRODUCCIÓN}

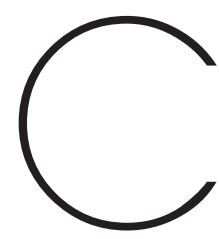

uando escuchamos de la necesidad de reconocer en su singularidad a la mujer no debemos pasar por alto que se trata de un cuerpo sexuado que solo se define correctamente en la historia y en el enfoque de la teoría de género. Se trata de una construcción social dinámica en la que no está exento el cotidiano de la salud y el padecer. Una nosología casi exclusiva de la mujer es la fibromialgia, término acuñado por Gowers desde 1904; más que una enfermedad se trata de un síndrome integrado de signos y síntomas caracterizados por el dolor localizado en puntos específicos del cuerpo. De manera que la fibromialgia es una enfermedad crónica compleja que provoca dolor muscular generalizado, acompañado de fatiga por lo que está asociado con un mal descanso nocturno.

Su reconocimiento, ha estado rodeado incluso de dudas sobre su verdadera existencia al asignarle un carácter psicógeno (reumatismo psicógeno). Sin embargo, es una condición frecuente entre los 20 y 50 años; se dice que su prevalencia puede ser el 5\% del total de la población occidenta.? En un estudio realizado por Quintero y cols., ${ }^{2}$ médicos del ISSSTE, estiman que entre el 2 al 3\% la población mundial es afectada. En el IMSS Alfredo Covarrubias y cols. ${ }^{3}$ señalan que al menos 5\% de los usuarios atendidos en Unidades de Medicina Familiar presentan esta condición dolorosa.

Actualmente, no se conoce la causa de la fibromialgia, sin embargo, se ha asociado a la presencia de micro traumatismos musculares que alteran la percepción y conducción del dolor. Así como también a una desregularización del sistema nervioso periférico, disfunción neuro inmuno endocrína, reducción de la concentración sérica de serotonina, cortisol, melatonina y algunas citosinas. ${ }^{4,5}$

Se ha demostrado que los pacientes tienen una hiperactividad simpática de día y de noche por este síndrome. La prevalencia es mayor en mujeres, por lo que hace sospechar la relación del síndrome con la situación social e histórica de la mujer. El
Ministerio de Salud en España ha declarado que "existen evidencias en el campo de la investigación en salud en cuanto a los factores de riesgo, las manifestaciones clínicas, las causas, consecuencias y tratamiento de las enfermedades pueden diferir entre hombres y mujeres".

Dentro de los factores de riesgo se incluye la presencia del estrés emocional significativo; aun cuando los datos asociados a eventos traumáticos son aun contradictorios y poco definitivos. Trabajos neurofisiológicos han encontrado que existe una disminución del umbral de dolor que involucra tanto áreas emocionales como sensoriales e incluso la zona somato sensorial primaria y secundaria de las personas que padecen fibromialgia. ${ }^{3}$

El cuadro clínico de la fibromialgia suele agravarse con el esfuerzo físico, la fatiga, la humedad ambiental, cambios climáticos e incluso por algunos factores laborales que tiene que ver con la postura durante el trabajo?. Con frecuencia las personas con fibromialgia refieren "Ientitud mental", confusión al hablar o escribir, torpeza, tendencia a dejar caer las cosas y los síntomas cambian de manera irregular de un día al otro.

La fibromialgia no puede ser explicada desde un abordaje únicamente medico; ya que los factores emocionales, sociales y culturales se ajustan más al modelo psicosocial de la enfermedad crónica de Engel. $^{8}$

De manera que los factores sociales y psicológicos no solo se remiten al momento en que clínicamente se manifiesta la fibromialgia sino a un proceso que incluye factores: predisponentes, desencadenantes y estabilizantes. Los factores predisponentes incluyen las condiciones familiares desfavorables de la infancia y las experiencias adversas de esta etapa de vida

Los factores desencadenantes con frecuencia incluyen eventos estresantes como crisis vitales o acontecimientos críticos. Un ejemplo claro de estos eventos es el mubing laboral. 
Finalmente, los factores estabilizantes se refieren a aquellas condiciones que mantienen de forma crónica la condición estresante; un ejemplo lo podemos encontrar en la comorbilidad de la fibromialgia con la ansiedad y la depresión.

\section{Dolor y género}

Ruíz López, afirmo en una entrevista realizada para la televisión educativa que el género es un factor determinante en el dolor. Esta afirmación la sustenta en los hallazgos recientes de la medicina experimental reportada en la Revista (Pain) muestran (Moguil) que el género femenino tiene mayor predisposición a sentir dolor no solo por la sensación sino por la elaboración de la propia sensación en los circuitos superiores cerebrales. La cultura, sociedad y la experiencia previa contribuyen a la elaboración de la sensación de dolor. En esta contribuye la propia familia que es el núcleo en el que durante la infancia se realizan los primeros aprendizajes. En sentido opuesto el Plan de salud Andaluz de 2008, señala que si bien la fibromialgia se presenta con mayor frecuencia en las mujeres; no es exclusiva de estas y por lo tanto puede ser usada como una desigualdad de género. ${ }^{10}$

En el génesis 3:16 de la biblia"1 se hace alusión al dolor en la mujer "en gran manera multiplicaré tu dolor en el parto, con dolor darás a la luz tus hijos. Con todo tu deseo será para tu marido. Y él tendrá dominio sobre ti". Sin embargo, ahora sabemos que existe una diferencia fisiológica sustantiva del dolor entre la mujer y el hombre. Esta diferencia alcanza la construcción cultural e histórica que los hombres y mujeres desarrollan desde sus inicios más tempranos.

Para el caso de la fibromialgia el dolor no solo muestra diferencias en su elaboración sino en la consistencia social e histórica de su condición.

La manera en que la mujer conceptualiza el cuerpo y sus funciones está íntimamente relacionada con los ambientes culturales. El pensamiento femenino queda al descubierto y puede estudiarse a través del discurso. La cultura y sus teorías entonces reconocen las diferencias de hombres y mujeres para construir su mundo subjetivo; sin perder de vista que la trama femenina se encuentra inserta en la totalidad social y cultural. En un mundo predominantemente masculino la cultura femenina resulta francamente marginal. Sin embargo, la vivencia del dolor suele estar en el marco de la interpretación y construcción real, sin ninguna marginalidad. El dolor en la fibromialgia está dotado de significado; el estar en el mundo a través del cuerpo sexuado determina las experiencias de las mujeres, aprendidas a través de los distintos códigos, culturales y lingüísticos. ${ }^{12}$

La elevada incidencia de la fibromialgia en la mujer no solo tiene explicación neurofisiológica ya que existe una verdadera construcción cultural del dolor. La propia expresión genera una cierta tranquilidad que muchas veces enmascara la verdadera importancia que el dolor y en la calidad de vida de las mujeres.

\section{El cuidado en la fibromialgia}

El proceso de cuidado de la fibromialgia se sustenta en una visión integral tanto del cuadro patológico como de los cuidados en el uso de medicamentos, actividad física, masajes, estiramiento, higiene postural e higiene psicológica.

La terapia cognitivo conductual y las técnicas de relajación ocupan un lugar especial; ya que su aplicación ha demostrado la disminución del dolor. ${ }^{13}$

El síntoma principal de la fibromialgia es el dolor; siendo el motivo central de la demanda de cuidado. La rigidez generalizada, la sensación de edema acompañada de hormigueo para completar el cuadro. La terapia cognitivo-conductual se basa en rechazar los pensamientos distorsionados de la persona acerca de sí mismo; reestructurando los pensamientos presentes por otros de carácter constructivo.

La administración de analgésicos, musicoterapia, acupresión, el fomento del ejercicio, asesoría y apoyo emocional contribuye a ir avanzando en el cuidado. Finalmente, el apoyo emocional y la participación de la red familiar suelen completar un enfoque integral de la fibromialgia.

Enf Neurol Vol. 16. No. 1 enero - abril 2017 
Los cuidados estandarizados pueden orientar el cuidado de grupos con el padecimiento, pero se debe asumir una actitud abierta, tomar la mejor alternativa de cuidado donde se haga visible el papel central de los factores sociales e históricos que se relacionan con la violencia hacia la mujer y el relegamiento de su condición.

\section{Fibromialgia y ejercicio}

Existen evidencias del efecto positivo de la práctica deportiva, del ejercicio aeróbico en las manifestaciones de la fibromialgia, ${ }^{14,}{ }^{15}$ El ejercicio en sí mismo mejora su condición tanto en mujeres como en hombres; además de proporcionar un efecto positivo en el estado de ánimo relacionado con la incidencia de fibromialgia.

Para fines de este artículo el ejercicio es cualquier actividad física que se hace o práctica para mantener o conservar la salud. Incluye un estilo de vida activo y el ejercicio de manera rutinaria. ${ }^{16}$ En tanto que entrenamiento debe de entenderse como el conjunto de ejercicios físicos que se realizan para perfeccionar el desarrollo de una actividad y especialmente la práctica deportiva. El entrenamiento requiere de fortaleza y resistencia. ${ }^{16}$ El entrenamiento de fortaleza también es conocido como ejercicio aeróbico en oposición al llamado ejercicio anaeróbico. El entrenamiento de resistencia crea fortaleza y músculo.

La intensidad del ejercicio se entenderá entonces como la energía que se invierte en la realización de la acción o actividad. .7 Por otro lado es importante la hora del día y durante cuánto tiempo se practicara el ejercicio.

La mayor parte del personal de salud, que está familiarizado con la fibromialgia recomienda ejercicios aerobios suaves, como natación, aerobios de bajo impacto o bicicleta estática. En un estudio con 38 pacientes que padecían fibromialgia, se mostró una disminución definitiva del dolor y mejoría de los síntomas después de seguir un programa gradual de acondicionamiento aerobio.
De acuerdo con una revisión Cochrane, menciona que el entrenamiento con ejercicios de resistencia de dos a tres veces por semana y durante doce semanas puede ayudar a reducir de manera importante el dolor, los puntos más sensibles e incluso la depresión, lo que en general se traduce en una mejora visible así como del bienestar general de la mujer con fibromialgia. ${ }^{18}$

Es importante mencionar que la intensidad del entrenamiento con ejercicios aeróbicos tiene efectos benéficos ya que reduce el dolor, sin embargo, se debe tomar en cuenta que este se debe ir incrementando paulatinamente hasta llegar a un nivel normal o moderado. Cabe mencionar que si al realizar los ejercicios, los pacientes fibromiálgicos perciben un aumento de los síntomas, deben bajar la intensidad hasta que éstos se aminoren, por lo que se recomienda que este tipo de ejercicios se deben de realizar una vez al día, durante al menos 20 minutos.

El ejercicio físico de bajo impacto mecánico como el taichi, yoga, caminata o ejercicios en el agua se han recomendado como una de las terapias efectivas para el manejo de los pacientes con fibromialgia. ${ }^{19-22}$ Tanto la balneoterapia, ejercicios en piscina de agua caliente ${ }^{23-27}$ como el entrenamiento aeróbico en seco. ${ }^{28-35}$

Rocha y González recomiendan la práctica de ejercicio aeróbico de bajo impacto seguido de estiramientos de grupos musculares unas 3 veces por semana con una duración de 20 a 30 minutos. $^{36}$

Por su parte el Institud Ferran de Reumatología ${ }^{37}$ recomienda incluir: precalentamiento, ejercicio aeróbico suave que no debe empeorar el problema y secuencia de relajación; todo esto bajo supervisión. Comenzar con estiramiento de 10 a 15 segundos e incrementar poco a poco hasta 30-60 segundos. Lo normal es tardar alrededor de tres meses para lograr el objetivo. Un buen propósito es el caminar de 20 a 30 minutos en terreno llano tres veces por semana y tras un mes pasar a practicar la caminata cada día de manera continua; esto es sin interrupción.
12

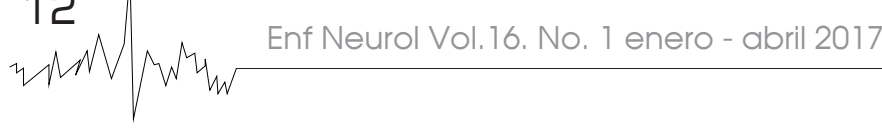


Tomar la precaución durante el ejercicio el de no aumentar la carga con abrigos u otros implementos (bolsas, botellas).

Alonso Alvares B. del Instituto de Rehabilitación Médica de Madrid en un estudio de revisión destaca que no existe un acuerdo único de los efectos del ejercicio de un programa concreto; no hay por lo tanto un entrenamiento único para todos los pacientes con diagnóstico de fibromialgial ${ }^{38}$ De manera que las pautas generales son: adoptar las pautas recomendadas por El Colegio Americano de Medicina Deportiva (ACSM, 1990), elegir ejercicio de fácil práctica diaria, siempre individualizar (intensidad y duración), Iniciar de forma progresiva, hacer pausas frecuentes, evitar trabajo excéntrico - ejercicio isométrico; incluir calentamiento y al final relajación; valorar las ventajas del ejercicio en grupos y considerar los efectos de la medicación en los pacientes antes de hacer cualquier indicación.

Así mismo se ha reportado que el entrenamiento con pesas tiene que ver con el sistema de aporte de oxígeno. Las personas con fibromialgia presentan un descenso en la oxigenación de tejidos; esto disminuye la síntesis de energía y el aporte al músculo, conduciendo a una fatiga rápida. Los capilares pueden tener grietas en las personas con fibromialgia. Esto puede ser la causa de la disminución del aporte de oxígeno.

De acuerdo con los estudios realizados en mujeres por Tomas Carus, los síntomas relacionados con la fibromialgia suelen mejorar con el ejercicio en agua. ${ }^{39}$ Sin embargo, en el mismo estudio no descarta el efecto placebo del programa de intervención.

Otra alternativa más es la alimentación sana, de acuerdo con la experiencia de la Asociación de Fibromialgia, Fatiga crónica y Síndrome Químico Múltiple de Navarra (AFINA), ya que esta contribuye a mejorar la calidad de vida. Por lo que esta asociación organiza talleres de cocina con el propósito de mejorar notablemente la condición de estos pacientes. ${ }^{40}$

\section{Consideraciones}

La práctica deportiva adecuada para cada mujer significa una estrategia viable para disminuir los síntomas de la fibromialgia.

Si bien el ejercicio mejora las manifestaciones clínicas, no todo ejercicio es válido para todos. Por lo que primero hay que hacer una evaluación individual, analizar qué es lo que genera el dolor y en función del origen hay que decidir qué ejercicios puede o no practicar. ${ }^{47}$

Hay que tomar en cuenta que la edad, en la que se presenta la fibromialgia (45-55 años), ya que a esta edad es frecuente que también estos pacientes presenten $y / O$ sufran otras enfermedades $\mathrm{y} / \mathrm{O}$ síndromes del aparato locomotor.

Por ejemplo, si la persona con fibromialgia padece además otras comorbilidades como:

Artrosis cervical se debe evitar los ejercicios repetitivos del cuello.

Lesión de la rodilla, sobrepeso: quizás los ejercicios más adecuados serán dentro del agua.

Hiperlaxo, la educación postural, realineación, isométricos será los más recomendable.

Contractura muscular, realizar ejercicios en piscinas de agua caliente o solo relajación dentro de ella para ir incrementando la actividad progresivamente.

El ejercicio físico es fundamental en la recuperación de estos enfermos, pero hay que tener en cuenta que los resultados no son inmediatos, sino después de varias semanas por lo que se debe continuar con la actividad física para no recaer.

El ejercicio se ha considerado como uno de los tratamientos no farmacológicos más eficaces en el manejo del dolor crónico y debilidad muscular ${ }^{42-46}$. Dentro de los beneficios que nos proporcionan son: Disminución del dolor, fatiga, aumenta la fuerza muscular, mejora la calidad del sueño, capacidad física, humor, estado psicológico, salud cardiorrespiratoria y calidad de vida.

Enf Neurol Vol. 16. No. 1 enero - abril 2017 . 13 


\section{REFERENCIAS BIBLIOGRÁFICAS}

1. Fibromialgia y deporte. Disponible en: http://deportesa/ud.com/deportesalud-fibromialgia-deporte.html (20 enero 2015).

2. Quintero G, Sánchez $\bigcirc$ y Rosas Barrientos. Frecuencia de la fibromialgia primaria y secundaria y patologías asociadas, en el servicio de Medicina de Rehabilitación del Hospital Regional 7ㅇ. De Octubre del ISSSTE. México. Disponible en: http://wuw.fibromialgia.nom.es/noticias-fibromialgia-sindromefatiga-cronica-sam-2009/frecuencia-de-la-fibromialgia-primaria-secundariay-patologias-asociadas-en-el-servicio-de-medicina-de-rehabilitacion. html (20 enero 2015)

3. Covarrubias Gómez A, Carrillo Torres O. Actualidades conceptuales sobre fibromialgia. Rev Mex de Anest 2016;39(1) 58-63.

4. Escolar Martin JM, Durán Barbosa R. Fisiopatología de la fibromialgia: alteraciones a nivel muscular y cerebral. Fisioterapia 2017:33(4):173-182.

5. Chávez Hidalgo D. Actualización en fibromialgia. Medicina Legal de Costa Rica 2013:30(1) Disponible en http://www.scielo.sa.cr/pdf/m/or/V30n1/ art08v30n1.pdt, consultado (5 diciembre 2016)

6. Sanidad. Fibromialgia. España, Ministerio de sanidad, política socia e igualdad. 2011. Disponible en: http://www.msssigob.es/profesionales/ prestacionesSanitarias/publicaciones/docs/fibromialgia.pdf, consultado (8 febrero 2015)

7. Blanco M, Arbonéz MM, Ruiz MJ. Evaluación de los aspectos psicológicos de la fibromialgia. España: Universidad de Girona, facultad de psicología. Disponible en: http://mww.psicolegs.org/Fotos/fm_virtu.pdf consultado (13 marzo 2015).

8. García-Bardón VF. La fibromialgia desde una perspectiva psicosocial: implicación de factores psicológicos. Hospital Universitario de Guadalajara hittp://fibromialgiamelilla wordpress.com/2014/08/24//a-fibromialgia-desdeuna-perspectiva-biopsicosocial-implicacion-de-factores-psicologicos/ consultado (10 marzo 2015)

9. Ruiz LR. El género es un factor determinante en el dolor: Dolor y género. Entrevista 2006.Disponible en: http://www.medicinatv.com/profesional/ videoteca/el-genero-es-un-factor-determinante-en-el-dolor-2022/dolor-ygenero-2 consultado (6 abril 2015)

10. Marón Morales R.., Cutilla Muños MA, Martínez Capa MR. Análisis enfermero de un Proceso de Atención Integrado: La fibromialgia. Tesis de Maestría Disponible en: https://books.google.com.mx/books?is.bn=1291028501, consultado (5 dic 2016).

11. La biblia. Génesis 3:16. Disponible en: http://bibliapara/ela.com/ genesis/3-16.htm consultado (19 febrero 2015).

12. Vivero Marín CE. El cuerpo como paradigma teórico en literatura Guadalajara: Rev. La ventana. 2008;3(28). Disponible en: http://mw . scielo.org.mx/scielo.php?pid=\$1405-94362008000200005\&script=sci_arttext consultado (19 febrero 2015)
13. Muñiz R, González Orta MLA, Nieto A. Cuidados de enfermería en pacientes con fibromialgia. Murcia: Enfermería Global. 2010:19. Disponible en: http://scielo.iscili.es/scielo.php?pid=S1695-61412010000200010\&script=sci arttext consultado (12 mayo 2015)

14. Santiago Latorre C, Torres Lacomba M. Fibromialgia y ejercicic terapéutico. Revisión sistemática cualitativa. Disponible en: http://coleporte rediris.es/revista/inpress/artfibromialgia776.pdf. consultado (18 abril 2015)

15. Brosseau L, Wells GA, Tugwell P, Egan M, Wilson KG, Dubouloz CJ, et al Ottawa Panel Members. Ottawa Panel evidence-based clinical practice guidelines for aerobic fitness exercises in the management of fibromyalgia part 1. PhysTher. 2008a:88(7):857-71

16. Actividad Física. Enciclopedia Médica. Medline Plus. Disponible en https://medlinep/us.gov/spanish/ency/article/001941.htm consultado (5 diciembre 2016)

17. The free diccionary by Farlex.Disponible en: $h$ ttp://es.thefreedictionary com/intensidad consultado (23 de septiembre 2015)

18. Busch AJ, Schachter CL, Overend TJ, Peloso PM, Barber KA. Exercise for fibromyalgia: a systematic review. J Rheumatol. 2008:35(6):1130-44

19. Carville SF, Arendt-Nielsen S, Bliddal H, Blotman F, Branco JC, Buskila D, et al. EULAR.EULAR evidence-based recommendations for the management of fibromyalgia syndrome. Ann Rheum Dis. 2008;67(4):536-41.

20. Brosseau L, Wells GA, Tugwell P, Egan M, Wilson KG, Dubouloz CJ, et al Ottawa Panel Members. Ottawa Panel evidence-based clinical practice guidelines for aerobic fitness exercises in the management of fibromyalgia part 1. PhysTher. 2008a;88(7):857-71

21. Mannerkorpi K, Iversen MD. Physical exercise in fibromyalgia and related syndromes. Best Pract Res ClinRheumatol. 2003:77:629-47.

22. Goldenberg DL, Burckhardt C, Crofford L. Management of fibromyalgid syndrome.JAMA. 2004;292(19):2388-95.

23. Jentoft ES, Kvalvik AG, Mengshoel AM. Effects of pool-based and landbased aerobic exercise on women with fibromyalgia/chronic widespread muscle pain. Arthritis Rheum 2001:45:42-7.

24. Gusi N, Tomas-Carus P, Häkkinen A, Häkkinen K, Ortega-Alonso A Exercise in waist-high warm water decreases pain and improves healthrelated quality of life and strength in the lower extremities in women with fibromyalgia. Arthritis Rheum. 2006:55(1):66-73.

25. Tomas-Carus P, Gusi N, Häkkinen A, Häkkinen K, Leal A, Ortega-Alonso A. Eight months of physical training in warm water improves physical and mental health in women with fibromyalgia: a randomized controlled trial. $J$ Rehabil Med. 2008;40(4):248-52
14

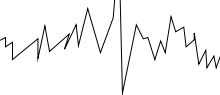
Enf Neurol Vol. 16. No. 1 enero - abril 2017 
26. Altan $L$, Bingol U, Aykac $M$, Koc $Z$, Yurtkuran $M$. Investigation of the effects of pool-based exercise on fibromyalgia syndrome. Rheumatol Int 2004:24:272-7.

27. Mannerkorpi K, Nyberg B, Ahlmén M, Ekdahl C. Pool exercise combined with an education program for patients with fibromyalgia syndrome: a prospective, randomized study. J Rheumatol. 2000;27:2473-87.

28. Wigers GH, Stiles TC, Vogel PA. Effects of aerobic exercise versus stress management treatment in fibromyal gia: a 4.5-year prospective study. Scand J Rheumatol. 1996:25:77-86

29. Meiworm L, Jakob E, Walker UA, Peter HH. Patients with flbromyalgia benefit from aerobic endurance exercise. Clin Rheumatol. 2000;19:253-7.

30. Jentoft ES, Kvalvik AG, Mengshoel AM. Effects of pool-based and landbased aerobic exercise on women with fibromyalgia/chronic widespread muscle pain. Arthritis Rheum. 2001:45:42-7.

31. Valim V, Oliveira L, Suda A, Silva L, de Assis M, Barros T, et al. Aerobic fitness effects in fibromyalgia. J Rheumatol. 2003;30(5): 1060-9.

32. Van Santen M, Bolwijn P, Verstappen F, Bakker C, Hidding A, Houben $H$, et al. A randomized clinical trial comparing fitness and biofeedback training versus basic treatment in patients with fibromyalgia. J Rheumatol. 2002a;29:575-81

33. Van Santen M, Bolwijn P, Landewe R, Verstappen F, Bakker C, Hidding A, et al. High or low intensity aerobic fithess training in fibromyalgia: does it matter? J Rheumatol. 2002b:29:582-7.

34. McCain GA, Bell DA, Mai FM, Halliday PD.A controlled study of the effects of a supervised cardiovascular fitness training program on the manifestations of primary fibromyalgia. Arthritis Rheum. 1988:31:1735-4.

35. Redondo JR, Justo CM, Moraleda FV, Velayos YG, Puche JJ, Zubero JR, et al. Long-term efficacy of therapy in patients with fibromyalgia: a physical exercise-based program and a cognitive behavioral approach. Arthritis Rheum. 2004:51:184-92

36. Rocha Ortiz M., Benito González E. La fibromialgia: Fundamentos y tratamiento. Rev Facultad de Ciencias de la Salud, Universidad Alfonso X el Sabio. 2006:4 http://wmw.uax.es/publicacion/fundamentos-y-tratamientode-la-fibromialgia.pdf consultado (2 septiembre 2015).
37. Intitud Ferran de Reumatologia. Un programa de ejercicio para e enfermo con fibromialgia. Disponible en: http://wmw.institutferran.org/ documentos/ejercicio_fm_ifr.pdf consultado (4 de septiembre 2015)

38. Álvarez B. A. Ejercicio físico en fibromialgia. Rehabilitación 2003; 37(6) 363-364

39. Tomas Carus P." Gusi Narcís, Leal A., García Yolanda, Ortega Alonso A. El tratamiento para la fibromialgia con ejercicio físico en agua caliente reduce el impacto de la enfermedad en la salud física y mental de mujeres afectadas. Reumatol Clin. 2007:3(7):33-7.

40. AFINA. Talleres de alimentación. Navarra: disponible en: http:/ fibromialgiaysalud.blogspot.mx/ consultado (18 abril 2015)

41. Munguía I. D., Legaz A. A., Alegre De M. C., Guía de práctica clínica sobre el síndrome de fibromialgia para profesionales de la salud, Edit. Elsevier España, S.A. Madrid, España, 2007:1-205.

42. Carville SF, Arendt-Nielsen S, Bliddal H, Blotman F, Branco JC, Buskila D et al. EULAR evidence-based recommendations for the management of fibromyalgia syndrome. Ann Rheum Dis. 2008;67(4):536-47.

43. Busch AJ, Schachter CL, Overend TJ, Peloso PM, Barber KA. Exercise for fibromyalgia: a systematic review. J Rheumatol. 2008;35(6):1130-44.

44. Brosseau L, Wells GA, Tugwell P, Egan M, Wilson KG, Dubouloz CJ, et al Ottawa Panel Members. Ottawa Panel evidence-based clinical practice guidelines for aerobic fitness exercises in the management of fibromyalgia part 1. Phys Ther. 2008a;88(7):857-71

45. Turk DC, Monarch ES, Williams AD. Psychological evaluation of patients diagnosed with fibromyalgia syndrome: a comprehensive approach. Rheum Dis Clin North Am. 2002:28:219-33.

46. Maquet D. Demoulin C, Croisier JL, Crielaard JM. Benefits of physica training in fibromyalgia and related syndromes. Ann Readapt Med Phys 2007:50(6):363-8, 356-62 